\title{
Gifts and Grants to ffPS
}

ffPS is most grateful for the gifts of $£ 25$ and over listed below, and also for many smaller donations, between 1st December 1981 and 31st March 1982.

\section{Oryx $100 \%$ Fund}

Elvetham Charitable Trust

Mrs J. Lyster

M.G. Gotts

Lady McNeice

E.D. Medcalf

J.M. Eckersley

Anonymous

L. Samiloff

G.C. Stoneham

K. Richards

D.J. Morris

S.K. Sharma

J.A. Collins

S.S. Johnson
$£$

500.00

382.00

100.00

100.00

100.00

50.00

50.00

44.00

40.00

35.00

30.00

30.00

25.00

25.00

\section{Mountain Gorilla Fund}

G.E. Gouley $(\$ 1000.00)$

J. Russell

Mr Ager

G.A. Vause

J.R. Sheppard

Wick School Fund

J. Vettier

G.C. Dunsterville

P.J. Sharp

\section{Bat Projects}

Dr R.E. Stebbings

Marwell Zoological Society

Monk Seal Appeal

A. Akers-Douglas

320.00

Bruce Coleman

J.J. Haines

Anonymous

Anonymous

Greek Animal Welfare

J.G, Phillips

M. Emerson

S.L. Jubic

Misc. and Anon. donations

200.00

100.00

100.00

50.00

50.00

36.00

25.00

25.00

6179.30

\section{Gifts}

New Moorgate Trust

1000.00

Ofenheim Charitable Trust

800.00

Major Lipscomb Memorial

269.00

M.M.T. Computing

100.00

200.00

T. Lilley Memorial Trust

100.00

52.00

Mr Bellis

50.00

50.00

40.00

Mr Henshall (Wadham College)

50.00

J.A. Rice

50.00

36.00

Mr Wohlwill

50.00

35.00

A. Ahlers

30.00

30.00

25.00

Grieveson, Grant and Co.

Charitable Trust Fund

25.00

\section{Oryx Endowment Fund}

100.00 Miss M.E. Gibbs

1000.00

25.00

Conder Conservation Trust 500.00

\section{Oryx $100 \%$ Fund Grants}

The following 100\% Fund grants were approved at the January 1982 Council meeting:

$£ 500$ to Sabina Knees for her study of the world trade in mahogany.

$\mathbf{f 2 5 0}$ to the Uganda National Parks to help finance two representatives to attend the New Zealand IUCN Conference. 